\title{
STATUS AND FUTURE PLANS OF THE PEP-II B-FACTORY*
}

\author{
Y. Cai, J. Clendenin, F. J. Decker, M. Donald, S. Ecklund, R. Erickson, A. Fisher, S. Heifets, \\ R. Holtzapple, R. Iverson, P. Krejcik, A. Kulikov, J. Nelson, A. Novokhatski, I. Reichel, \\ J. T. Seeman, M. Stanek, M. Sullivan, J. Turner, U. Wienands, SLAC, Stanford, CA 94309, USA \\ C. Steier, M. Zisman, LBNL, Berkeley, CA 94720, USA \\ M. Biagini, INFN, Frascati, Italy \\ W. Kozanecki, Saclay, France
}

\begin{abstract}
The PEP-II e+e- collider [1,2,3,4] has been operating for two years with the BaBar detector at the energy of the Upsilon $4 \mathrm{~S}$ resonance. The peak luminosity has reached $3.3 \times 10^{33} / \mathrm{cm}^{2} / \mathrm{s}$ with 693 bunches with a positron current of $1.5 \mathrm{~A}$ and an electron current of $0.8 \mathrm{~A}$. PEP-II has delivered in excess of $38 \mathrm{fb}^{-1}$ of data to BaBar. The beambeam tune shift limits are approaching 0.05-0.07 horizontally and 0.03-0.05 vertically [5]. The electron cloud instability ECI enlarges the positron beam size at high currents but is reduced by a solenoidal field on the vacuum chambers [6]. The beam currents in PEP-II are being raised to increase the number of bunches and the luminosity. Over the next few years the luminosity goal for PEP-II is $10^{34} / \mathrm{cm}^{2} / \mathrm{s}$.
\end{abstract}

\section{PEP-II COLLIDER}

PEP-II is an e+e- collider with asymmetric energies (3.1 and $9 \mathrm{GeV}$, respectively) in a $2200 \mathrm{~m}$ tunnel at the Stanford Linear Accelerator Center. The collider produces B mesons to study a particle physics effect called CP violation as well as other physics topics. PEP-II was completed in 1998 with the first luminosity generated in July of that year. The installation of the BaBar Detector was finished in May 1999.

The overall layout of PEP-II is shown in Figure 1. The accelerator parameters and achievements of the High Energy Ring (HER) are listed in Table 1 and those for the Low Energy Ring (LER) in Table 2. The two beams collide at a single point in the IR2 hall where the BaBar detector is located. Beam parameters at the best luminosity are shown in Table 3. General PEP-II milestones are listed in Table 4.

The salient features of the PEP-II collider design are LER-above-the-HER rings, head-on collisions but with dipoles bends near the collision point, new copper RF cavities with HOM damping, strong bunch-by-bunch feedback systems, many bunches (1658), high stored charges $(2.0 \mathrm{~A} \times 1.0 \mathrm{~A})$, permanent magnet interaction region quadrupoles and dipoles, and use of the existing SLAC linac as an injector but with new transport lines.

\footnotetext{
"Work supported in Department of Energy contract numbers DE-AC0376SF00515 and DE-AC03-76-SF00098.
}

The PEP-II run with BaBar is going very well. The luminosity reached $110 \%$ of design or $3.3 \times 10^{33} / \mathrm{cm}^{2} / \mathrm{s}$ on October 29, 2000, and an integrated luminosity day of $163 \%$ of design or $220 \mathrm{pb}^{-1}$ occurred June 29, 2001 The total delivered integrated luminosity to date is $38 \mathrm{fb}^{-1}$. The peak luminosity and integrated luminosity plots over the past two years are shown in Figures 2 and 3.

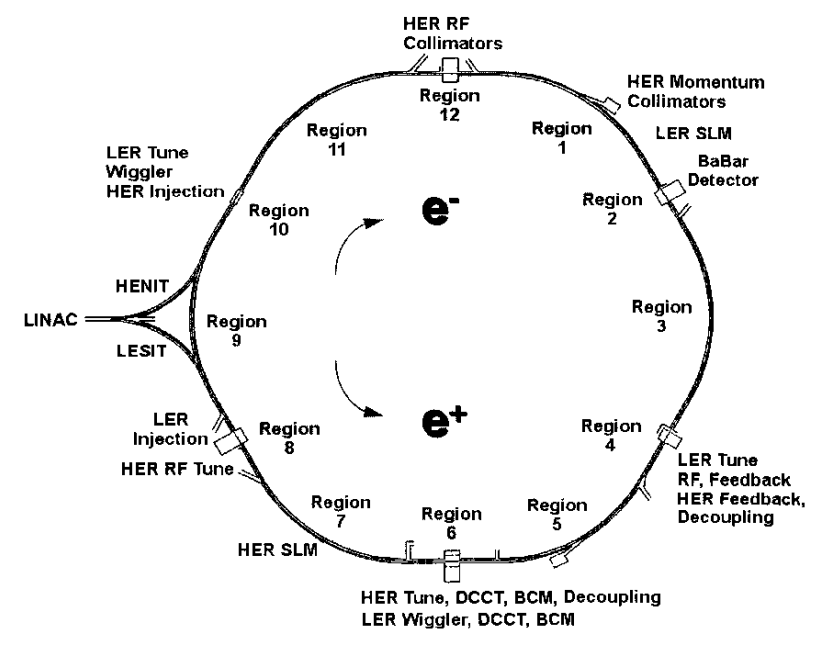

Figure 1: PEP-II B-Factory Overview

Table 1 PEP-II HER Parameters and Results

$\begin{array}{lll}\text { HER Parameter } & \text { Design } & \text { Achieved } \\ \text { Beam energy }(\mathrm{GeV}) & 9.0 & 9.1 \\ \text { Number of bunches } & 1658 & 1658 \\ \text { Particles per bunch } & 2.1 \times 10^{10} & 5.6 \times 10^{11} \\ \text { Total current }(\mathrm{mA}) & 750 & 920 \\ \text { Current per bunch }(\mathrm{mA}) & 0.45 & >12 . \\ \text { Bunch spacing }(\mathrm{m}) & 1.26 & 0.63 \\ \text { Bunch length }(\mathrm{mm}) & 11.0 & 12.0 \\ \Delta \text { E/turn }(\mathrm{MeV}) & 3.6 & 3.6 \\ \mathrm{RF} \text { frequency }(\mathrm{MHz}) & 476 . & 476 . \\ \text { Ion clearing gap }(\%) & 5 & 5 \\ \text { RF voltage }(\mathrm{MV}) & 14.0 & 14.0 \\ \text { Rel. energy spread }\left(10^{-3}\right) & 0.61 & 0.61 \\ \text { Synchrotron tune } & 0.045 & 0.045 \\ \text { Betatron tune }\left(\mathrm{v}_{\mathrm{x}} / \mathrm{v}_{\mathrm{y}}\right) & 24.62 / 23.64 & 24.57 / 23.64\end{array}$


Table 2 PEP-II LER Parameters and Results

$\begin{array}{lll}\text { LER Parameter } & \text { Design } & \text { Achieved } \\ \text { Beam energy }(\mathrm{GeV}) & 3.1 & 3.1 \\ \text { Number of bunches } & 1658 & 1658 \\ \text { Particles per bunch } & 5.9 \times 10^{10} & 3.2 \times 10^{11} \\ \text { Total current }(\mathrm{mA}) & 2140 & 2141 \\ \text { Current per bunch }(\mathrm{mA}) & 1.29 & >7 . \\ \text { Bunch spacing }(\mathrm{m}) & 1.26 & 0.63 \\ \text { Bunch length }(\mathrm{mm}) & 10.0 & 12 . \\ \Delta \text { E/turn }(\mathrm{MeV}) & 0.75 & 0.68 \\ \text { RF frequency }(\mathrm{MHz}) & 476 . & 476 . \\ \text { Ion clearing gap }(\%) & 5 & 5 \\ \text { RF voltage }(\mathrm{MV}) & 5.1 & 4.5 \\ \text { Rel. energy spread }\left(10^{-3}\right) & 0.77 & 0.70 \\ \text { Synchrotron tune } & 0.033 & 0.032 \\ \text { Betatron tune }\left(\mathrm{v}_{\mathrm{x}} / \mathrm{vy}\right) & 38.57 / 36.64 & 38.65 / 36.58\end{array}$

\section{ACCELERATOR PHYSICS}

The peak luminosity was achieved using 693 bunches with $1550 \mathrm{~mA}$ of positrons and $850 \mathrm{~mA}$ of electrons. The bunches were spaced every four RF buckets in 35 short trains of 20 bunches each. There is a 5\% ion clearing gap. The beta $\mathrm{x}$ and $\mathrm{y}$ at the collision point were $50 \mathrm{~cm}$ and $1.25 \mathrm{~cm}$, respectively. The first $\mathrm{e}^{+}$bunch in each minitrain is under filled by about $10 \%$ to avoid blowing up its respective $\mathrm{e}^{-}$bunch by the beam-beam force. That $\mathrm{e}^{+}$ bunch is not enlarged as much by ECI as the other $\mathrm{e}^{+}$ bunches.

Table 3 PEP-II Collision Parameters

\begin{tabular}{|c|c|c|}
\hline IR Parameter & Design & Present Operating \\
\hline C-M energy (GeV) & 10.28 & 10.28 \\
\hline Crossing angle (mrad) & 0.0 & $<0.1$ \\
\hline Luminosity $\left(\times 10^{33}\right)$ & 3.00 & 3.30 \\
\hline Number of bunches & 1658 & 693 \\
\hline HER current (mA) & 750 & 850 \\
\hline LER current (mA) & 2146 & 1550 \\
\hline \multicolumn{2}{|c|}{ Beam-beam parameter $(y+/-) 0.03$} & $0.055 / 0.028$ \\
\hline \multicolumn{2}{|c|}{ Beam-beam parameter $(x+/-) 0.03$} & $0.069 / 0.059$ \\
\hline$\beta \mathrm{y} * / \beta \mathrm{x} *(\mathrm{~cm} / \mathrm{cm})$ & $1.5 / 50$ & $1.25 / 50$ \\
\hline Optimum coupling (\%) & 3.0 & 2 to 6 \\
\hline Emittance (nm-rad) (y/x) & $1.5 / 49$. & $3 / 30+, 2 / 50$ \\
\hline IP rms beam $\sigma_{\mathrm{y}} / \sigma_{\mathrm{x}}(\mathrm{mm})$ & 4.7/157. & $5.0 / 147$ \\
\hline$\sum_{\mathrm{x}, \mathrm{y}}($ microns) $($ at low I) & $6.7 / 222$ & $6.7 / 207$ \\
\hline Injection top-off time (min) & & 2 \\
\hline Injection full fill time (min) & & 8 \\
\hline Detector solenoid field (T) & 1.5 & 1.5 \\
\hline Int. luminosity in 8 hours pl & $\mathrm{pb}^{-1} \quad 45$ & 76 \\
\hline Int. luminosity in 24 hours & $\mathrm{pb}^{-1} 135$ & 220 \\
\hline Int. luminosity per week pb & $b^{-1} \quad 785$ & 1048 \\
\hline Int. luminosity per month $\mathrm{p}$ & $\mathrm{pb}^{-1} \quad 3300$ & 3870 \\
\hline Total integrated luminosity & $y$ thru $6 / 22 / 0$ & $38 \mathrm{fb}^{-1}$ \\
\hline
\end{tabular}

Table 4 PEP-II Milestones

\begin{tabular}{|c|c|c|}
\hline Date & & Milestone or Commissioning \\
\hline 1994 & January & Start of PEP-II construction \\
\hline 1997 & June 16 & HER beam first stored \\
\hline 1998 & July 16 & LER beam first stored \\
\hline 1998 & July 23 & First collisions \\
\hline 1999 & May 10 & BaBar detector installed \\
\hline 1999 & May 26 & First hadronic events recorded \\
\hline 1999 & June 15 & Scan of the Upsilon $4 \mathrm{~S}$ \\
\hline 1999 & Sept 14 & Luminosity peak of $1.35 \times 10^{33}$ \\
\hline 2000 & June 11 & Integrated $150 \mathrm{pb}^{-1}$ in one day \\
\hline 2000 & Oct 29 & Luminosity peak of $3.30 \times 10^{33}$ \\
\hline 2001 & June 29 & Integrated $220 \mathrm{pb}^{-1}$ in 24 hours \\
\hline 2001 & June 29 & Total delivered luminosity of $38 \mathrm{fb}^{-1}$ \\
\hline
\end{tabular}

Several coasting beam fills with BaBar were tried with a bunch pattern having every other bucket filled. This bunch spacing is the design pattern (called the "by-2" pattern) having potential parasitic crossing effects. With full colliding currents and the by- 2 bunch pattern the nominal peak luminosity was nearly achieved (90-95\%) in a few hours. The main effect was to shift the vertical tune by about 0.01 . This bodes well for the future when we will try for higher luminosity with more bunches.

Over the past year, a PEP-II vacuum bellows next to the beryllium vacuum chamber in the center of BaBar has developed elevated temperatures at the higher beam currents. Studies were made of the heating of this component under various beam conditions (current, bunch length, number of bunches, orbit offsets and angles). It is likely that the heating comes from HOM power from an abrupt transition crotch in the forward end of the IR.

The transverse size of the positron beam is enlarged at high currents by the electron cloud instability from multipacting electrons, as is now commonly seen in several accelerators. A solenoid capable of 30 gauss has been wrapped on the straight section vacuum chambers to suppress these electrons. A length of $600 \mathrm{~m}$ has been wound resulting in a luminosity increase of order $70 \%$ over the past year. With the present solenoids the beam size enlargement starts at about $1300 \mathrm{~mA}$ with four bucket spacing and is seen in both the vertical and horizontal planes. PEP-II is now embarking on a program to wind solenoids in the LER bending arcs to suppress multipacting and photo-electrons there. About 200 chambers $(5 \mathrm{~m}$ each) need winding. $80 \mathrm{~m}$ is done so far.

The LER wiggler was turned off a year ago last February to reduce the LER beam size at the collision point to increase the luminosity. The luminosity increased about $40 \%$. Most of the loss in luminosity with the wiggler on was traced to a strong induced $\mathrm{x}-\mathrm{y}$ coupling. Nothing in the wiggler magnetic measurements data would indicate such a strong coupling. In addition to generating radiation, the wiggler changes the orbit length due to the trajectory wiggles and, thus, moves the beam orbit radially over all of LER. As a test during the 
machine studies, a similar radial orbit shift was made by changing the RF frequency while looking at the $x-y$ coupling. The lattice as designed should not couple the beams with a radial orbit shift. However, the RF frequency shift did change the coupling and about as much as the wiggler did. The coupling was traced to a changing vertical orbit in the interaction region which changes the beam position in the local sextupoles causing coupling.

The beams at the interaction point were measured to be tilted by about 1.5 degrees relative to the machine axis. There were also hints from the out of plane beam-beam deflection scans that the two beams were slightly tilted relative to each other.

Tests were made to center the minima of the IR y beta functions at the collision point by moving the waists with the IR quadrupoles while measuring the luminosity using the rf relative phase. The waists were found to be very nearly centered to a few $\mathrm{mm}$.

\section{PEP-II UPGRADES}

In the three month 2000-2001 winter downtime, new equipment was installed to improve the performance capability of PEP-II. The third LER RF station was installed to allow the LER current to be raised from $1.8 \mathrm{~A}$ to 2.7 A. Two new LER movable collimators have been installed near the interaction point to improve the BaBar backgrounds at high currents. A shielding wall was installed in the PEP-II tunnel upstream of BaBar to remove "tunnel shine" from affecting the IFR detectors. Additional vacuum pumps were added to LER Arc 7 to improve the vacuum pressure. A new HER vacuum chamber from 10 to $20 \mathrm{~m}$ upstream of BaBar was installed to lower the average pressure to well below 1 nTorr. A new HER high power dump was installed. All these tasks were successful commissioned in spring 2001.

Several upgrade projects for summer 2002 have been started aiming at higher beam currents and an increased luminosity. Two new rf stations (fourth LER and sixth HER) have been started. These new stations will allow the

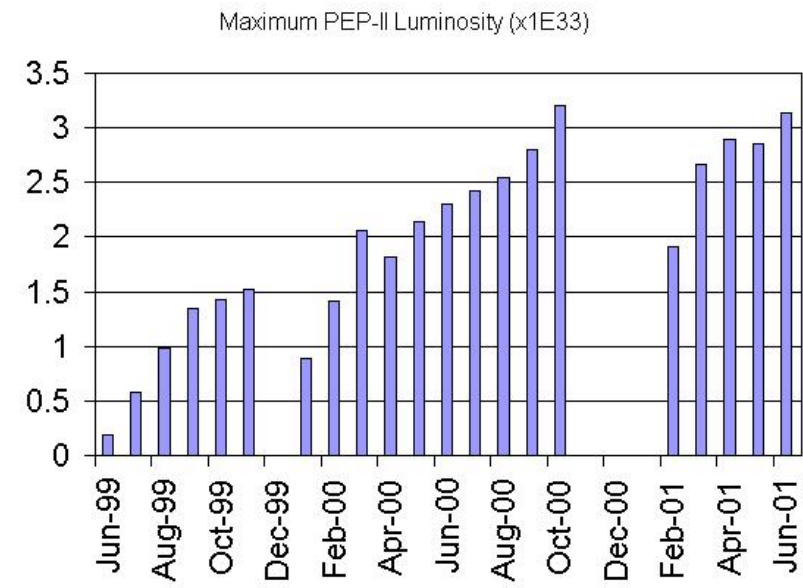

Figure 2 Peak luminosity in PEP-II during each month.
PEP-II Monthly Integrated Luminosity

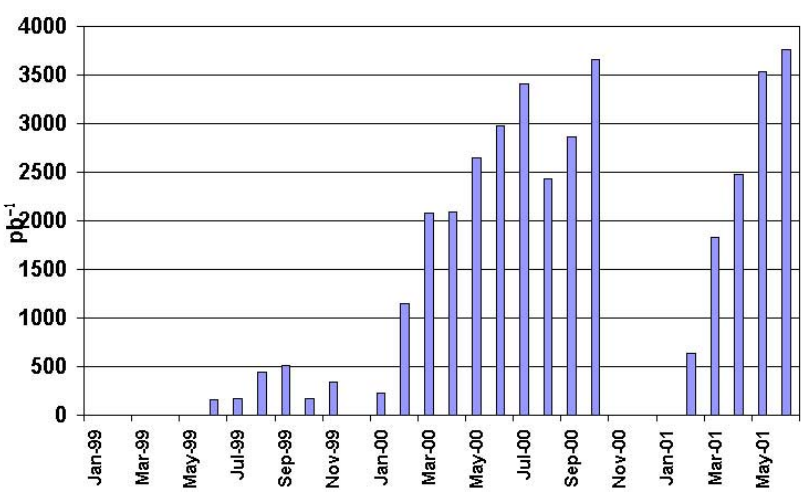

Figure 3: Monthly integrated luminosity delivered by PEP-II to BaBar.

currents to be raised by $20-30 \%$ in each ring. Construction has started on improved interaction region vacuum chambers to reduce the HOM heating near the IR.

\section{PEP-II FUTURE PLANS}

PEP-II is to run continuously through to the end of June 2002 with the hope of integrating $100 \mathrm{pb}^{-1}$. Then a three to four month down is needed to replace several vacuum chambers near the IR, install two new RF stations, and to repair the Instrumented Flux Return IFR of BaBar.

There are several longer range upgrade plans in place to raise the luminosity to $10^{34}$ and, furthermore, the issues for $2 \times 10^{34}$ are under study. Several of the issues include understanding the empirical skew quadrupole adjustments to make smaller spot sizes, raising the LER and HER currents, reducing beam aborts from short radiation bursts in $\mathrm{BaBar}$ and from the RF system, lowering beta $\mathrm{y}^{*}$ from $1.25 \mathrm{~cm}$ to $0.7 \mathrm{~cm}$, reducing the electron cloud instability, replacing several hot vacuum chambers, and raising the number of bunches.

\section{REFERENCES}

[1] "PEP-II Conceptual Design Report", SLAC Report 418, LBL-PUB-5379, June (1993).

[2] F.J. Decker, et, al, "Multibunch Operation of PEPII", EPAC 2000, Vienna, Austria.

[3] Y. Nosochkov, et al, "Recent PEP-II Lattice Developments', EPAC 2000, Vienna, Austria.

[4] J. Seeman et al., "Status Report on PEP-II Performance," EPAC 2000, Austria.

[5] Y. Cai et al, "Beam-Beam Measurements in PEP-II," Proc. PAC 2001.

[6] A. Kulikov et al., "ECI in PEP-II LER," Proc. PAC 2001. 